Die Anatomie der Umbellif erenachse in ihrer Beziehung zum System. 1895.

为

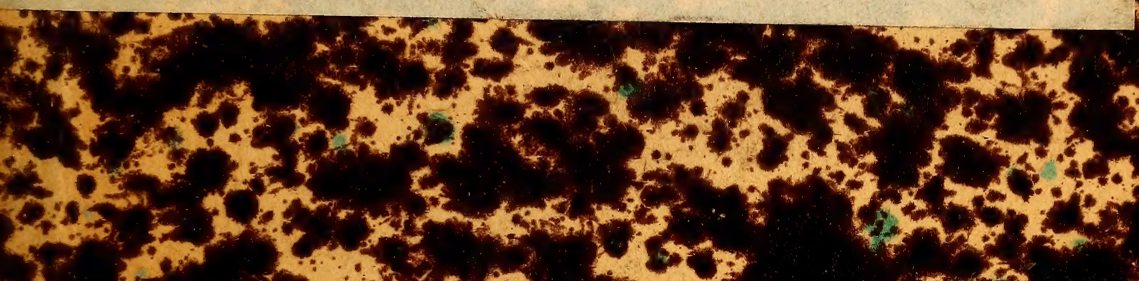

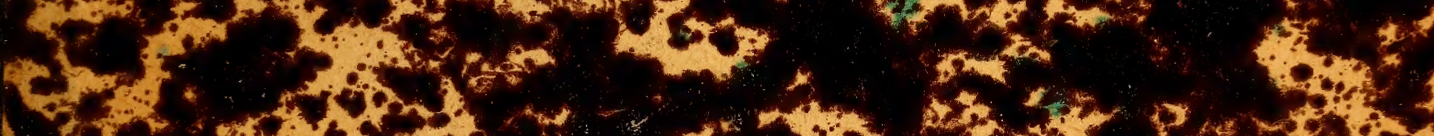

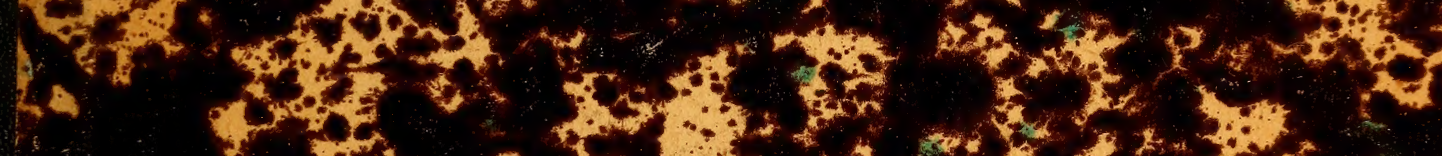

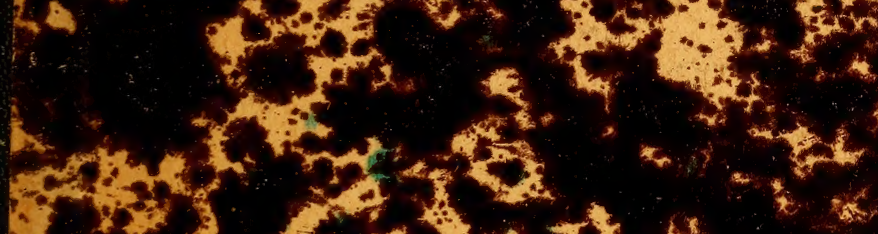

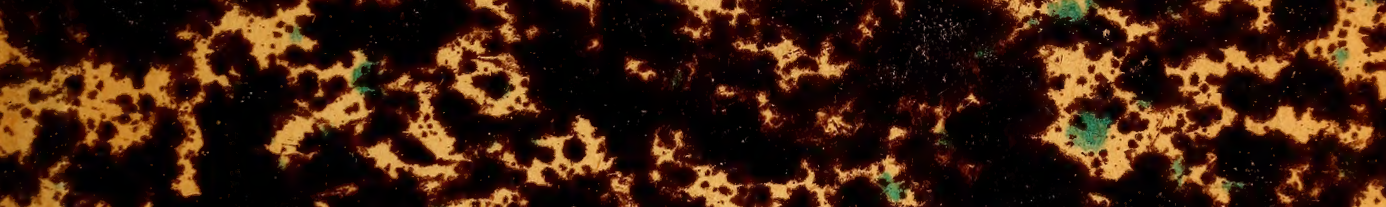

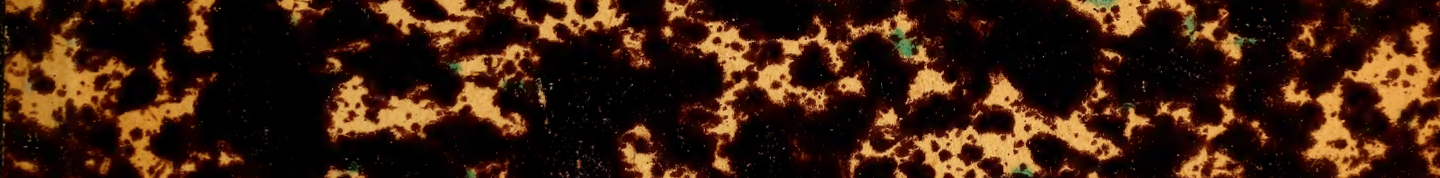

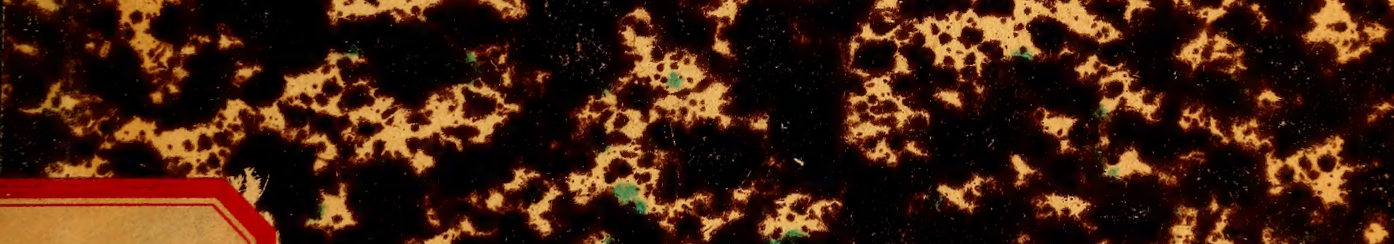

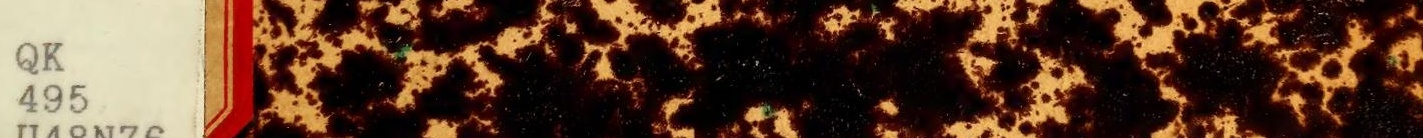
U95 4876
1895
Bot.

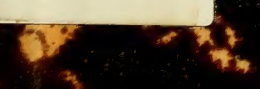
tistis

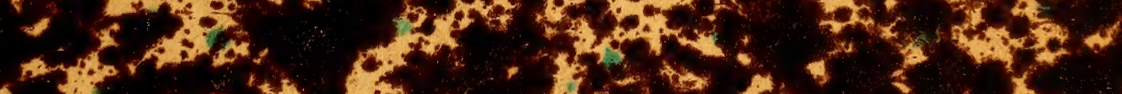

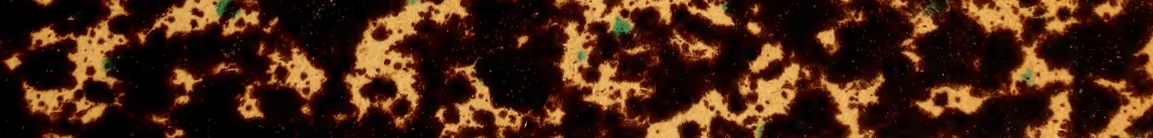
? $+40.2 x^{2}$ a. 



\section{Die Anatomie der Umbelliferenachse in ihrer Beziehung zum System.}

\section{Inaugural-Dissertation}

zur

Erlangung der Doktorwürde der

hohen philosophischen Fakultät

der

Friedrich-Jilexanders- đaniversifäf Errlangen vorgelegt von

\section{F. van Noenen} aus Gểdern.

Tag der mündlichen Prüfung: 30. Januar 1895.

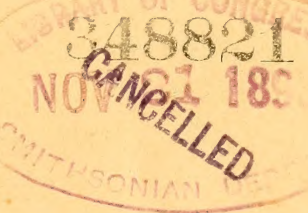

Erlangen.

K. b. Hofbuchdruckerei von Aug. Vollrath. 1895. 
Gedruckt mit Genehmigung der philosophischen Fakultät zu Erlangen.

Referent: Prof. Dr. Max Reess. 
$. N>6$

\section{Seinen feuren Elfern} in

Isiebe und Dankbarkeil gewidmet

vom Verfasser. 



\section{Einleitung.}

Die Umbelliferen bilden durch ihre morphologischen Eigentümlichkeiten im Rahmen des natürlichen Systems eine ausserordentlich gut charakterisierte, in sich vollständig geschlossene Familie. Sieht man von der verhältnismässig kleinen Anzahl von Species der Triben Hydrocotyleae, Saniculeae und Mulineae ab, welche den allmählichen Übergang zu den typischen Formen der Familie bilden, so findet man, dass sich nur auf Grund einiger, meist wenig in die Augen fallenden Modifikationen des Fruchtbaues eine natürliche Einteilung gründen lässt. Die Zusammengehörigkeit aller heute als Umbelliferen geltenden Arten wurden deshalb auch von den ersten zusammenfassenden Systematikern schon anerkannt und ist bis auf die neusten Forscher in ihren diesbezüglichen Systemen bestätigt worden.

Die innere Gliederung der Familie in die Unterfamilien: Orthospermeae, Campylospermeae und Coelospermeae hat man in jüngster Zeit vielfach fallen gelassen und statt dieser die Einteilung in Heterosciadieae, Haplozygieae und Diplozygieae vorgezogen, eine Modifikation, welche sich auch anatomisch rechtfertigen lässt, wenigstens was die Trennung der Heterosciadieae von den beiden übrigen Unterfamilien anlangt. Im übrigen hat das Umbelliferensystem wenig Umwandlung durchgemacht.

Da nun für die Systematik die sogen. anatomische 
Methode zu so grosser Bedeutung gelangt ist und in den letzten Jahren speciell durch Engler und Radlkofer sich allgemeine Anerkennung zu verschaffen gewusst hat, war ich an die Aufgabe herangetreten die Familie der Umbelliferen, besonders den Stengel, anatomisch durchzuarbeiten in der Erwartung, dass man die Triben, Gattungen oder gar die Species anatomisch würde unterscheiden können. Ob dieses unter Zugrundelegung der anatomischen Verhältnisse auch der übrigen Organe jemals möglich sein wird, lasse ich dahingestellt sein. Für die Umbelliferen haben meine Untersuchungen jedenfalls dargethan, dass die Struktur der Achse zur Unterscheidung der Triben schon nicht mehr ausreicht, geschweige denn um die mehr als 1300 Arten zu unterscheiden.

Die folgenden Untersuchungen werden die Zweigstruktur der Umbelliferen näher kennen lehren und zeigen, inwiefern Relationen zwischen Morphologie und Histologie bei ihnen vorhanden sind.

Was das Untersuchungsmaterial anbelangt, so entstammt dasselbe zum grössten Teil dem Herbarium des Herrn Apothekers Reiss-Lüdinghausen. Dasselbe zeichnet sich durch seine grosse Reichhaltigkeit und musterhafte Ordnung aus, und kann das hier aufgespeicherte Material wohl durchgehends als richtig bestimmt gelten. Ausserdem stand mir das hiesige Universitätsherbar zur Verfügung, aus welchem ich hier und da zur Vervollständigung des Materials geschöpft habe.

Herrn Prof. Dr. Reess, der mir die Anregung zur Arbeit gab und mich bei Ausführung derselben mit Rat und That zur Seite stand, bin ich zu grossem Dank verpflichtet. Ferner sei es mir gestattet an dieser Stelle auch Herrn Reiss für die gütige Überlassung seines Herbars meinen besten Dank auszusprechen. 


\section{Allgemeiner Teil.}

\section{Die verschiedenen Querschnittstypen.}

Sieht man von den Zellen und ihren metamorphosierten Derivaten, aus welchen sich die Achse der Umbelliferen aufbaut, an und für sich ab, und betrachtet man nur die Konstellation der verschiedenen Gewebsarten zu einander, so kann man für dieselben vier Typen aufstellen, unter welchen fast alle Gattungen und Arten zusammengefasst werden können.

Der Typus, dem der weitaus grösste Teil der Familie entspricht, zeigt im grossen und ganzen folgende Zusammenstellung.

Der einzelligen Epidermis folgt, alternierend mit dem Chlorophyllparenchym, eine mehr oder weniger starke Collenchymplatte. Diesen folgt das Rindengewebe und diesem der Gefässbündelring, dessen einzelne Bündel durch eine Strangscheide meist ununterbrochen mit einander verbunden sind, zuweilen aber auch primäre Markstrahlen zwischen sich lassen. Das Mark füllt entweder den vom Gefässbündelring umschriebenen Raum vollständig aus, oder es ist im Centrum zerrissen, und der Stengel ist dann hohl. Diese Zusammenstellung zeigen, wie schon oben erwähnt, der weitaus grösste Teil der Umbelliferen, und wird hierdurch auch der einheitliche Charakter der Familie, den sie in ihrem morphologischen Aufbau zeigt, bestätigt.

Ein zweiter Typus, der seine Vertreter auschliesslich in dem Tribus Hydrocotyleae findet, weicht wesent- 
lich von dem vorher beschriebenen dadurch ab, dass das collenchymatische Hypoderm wenig ausgebildet ist, zuweilen sogar vollständig verschwindet wie bei Dimetopia, und meist nur in Form einer einzelligen subepidermalen Schicht auftritt, so bei Hydrocotyle vulgaris, H. virgata, H. flexuosa, Didiscus coeruleus u. a. Endlich kommt es auch vor, dass in oder unter dem ein- oder zweizelligen Collenchymgewebe einzelne Zellen stark sclerotisch verdickt sind, welche daun auf dem Querschnitt eine den Umbelliferen sonst fremde Form darbieten. Bei gewöhnlichem Durchmesser sind sie kreisrund und ihr Lumen ist meist sehr eng, oft sogar vollständig verschwunden. Ausserdem laufen sie nicht wie die typischen Collenchymzellen nach oben und unten spitz zu, sondern sind an den Enden abgeflacht: Xanthosia rotundifolia, X. pilosa. Fig. 1 u. 2. Das Rindengewebe kann verschieden ausgebildet sein. Entweder besteht es bei einem eng zusammengedrängten axilen Strangsystem, wie bei den Hydrocotyleae-Species, aus einer breiten Schicht weitlumiger Elemente, oder es ist nur aus einigen wenigen Zellreihen zusammengesetzt, so dass es im Vergleich zu den übrigen Gewebekomplexen fast vollständig verschwindet, so bei Dimetopia. Die verbindenden Libriformbrücken der Strangscheide fehlen meist, doch bildet oft der Holzkörper durch Aneinanderrücken der Gefässbündel einen festen, geschlossenen Ring. Das Mark ist stets vollständig.

Als dritten Typus könnte man wohl den der monocotylenähnlichen Eryngien aufstellen. ${ }^{1}$ ) Dieselben zeigen als charakteristische Eigentümlichkeit neben anderen Merkwürdigkeiten Luftkanäle und Gefässbündel, welche das Rindengewebe durchziehen. Sie erhöhen

$\left.{ }^{2}\right)$ M. Mo ebius: Untersuchungen über die Morphologie und Anatomie der monoketylenähnlichen Eryngien. Pringsheim's Jahrbücher Bd. XIV pag. 379. 
auf diese Weise das Fremdartige, welches diese parallelnervigen Eryngien andern Dicotyledonen und speciell den Umbelliferen gegenüber schon durch ihren Habitus bieten noch durch diese anatomischen Eigentümlichkeiten.

Der nun folgende vierte Typus geht durch eine Reihe von Triben. Er umfasst alle die Species, welche zwar nach dem ersten Typus zusammengesetzt sind, ausserdem aber noch markständige Bündel zeigen.

Die Gattung Mulinum, deren Abnormitäten später eingehend beschrieben werden sollen, schliesst sich keinem der hier beschriebenen vier Typen an.

Kurze Anatomie der Umbelliferenachse. $\left.{ }^{1}\right)$

\section{Epidermis.}

Die Epidermis ist meist normal gebaut, d. h. ihre Zellen sind, wie fast überall am Stengel der Phanerogamen, auch hier vertikal gestreckt und meist hexagonal in der Oberflächenansicht.

Sie ist mit Ausnahme von Mulinum ferosa einschichtig. Bei dieser Species, die auch in anderer Beziehung vielfach Abnormitäten zeigt, begegnen wir einer zweischichtigen Epidermis. Eine erwähnenswerte Eigentümlichkeit zeigen ferner Peucedanum Chabraei und P. heterophylla. Bei der ersten Species besitzt die Membran und somit auch die cuticala sämtlicher Epidermiszellen mit Ausnahme derer, welche das Collenchym bedecken, nach aussen hin warzenähnliche F'ortsätze. Bei Peucedanum heterophylla findet man diese Abweichung auch, jedoch tritt sie hier nicht so constant bei allen Zellen auf, wie bei Chabraei. Fig. 3. Die Cuticularschicht ist im

1) Jochmann: De umbelliferarum structura et evolutione nonnulla. 1855. Courchet: Les ombellifères. Montpellier 1882. 
allgemeinen normal entwickelt, zuweilen ist sie schön regelmässig gewellt, wie bei Heracleum spondylium, H. alpinum, Spananthe paniculata, und bei Xanthosioa rotundifolia ist sie auffallend schön geschichtet.

Bisweilen treten durch Auswachsen einzelner Epidermiszellen Haare auf, die aber niemals gekammert sind. Bei Chaerophyllum elegans, Ch. cicutarium und Ch. aureum sind dieselben besonders gross ausgebildet und tritt noch hinzu, dass ihr Fuss von einer Rosette besonders gestalteter Nebenzellen ringförmig umgeben ist. Drüsenhaare und sonstige Trichombildungen kommen bei den Umbelliferen nicht vor.

\section{Die Rinde.}

In der grünen Rinde der Umbelliferen ist das Vorkommen von Collenchym typisch. Dasselbe füllt, auf dem Querschnitt betrachtet, stets die Ausbuchtungen, welche jedesmal einer Kante der Achse entsprechen, als mächtige Platte aus. Sie ist meist nierenförmig und mit ihrer concaven Fläche gegen das Innere des Stengels gewendet. Da die Zahl und Grösse der Kanten sehr variabel ist, variieren in Grösse und Zahl auch die Collenchymplatten. Der Raum zwischen ihnen wird von Chlorophyllparenchym ausgefüllt. Die hier beschriebenen Verhältnisse finden sich in der Rinde aller Umbelliferen vor, mit Ausnahme der meisten Arten von Hydrocotyleae und Saniculeae. Manchmal sieht man das Collenchym so stark auftreten, dass es einen fast ununterbrochenen Mantel bildet, der nur hier und da eine Lücke lässt, welche von Chlorophyllparenchym ausgefüllt ist, und der ausserdem noch in den Radien der grossen Gefässbündel eine ansehnliche Verstärkung erfahren hat, so bei Athamantha vestina und Laserpitium Nestleri. Bei andern Arten tritt das Collenchym wieder sehr zurück und bildet vor den Gefässbündeln nur eine kleine 
Platte, welche etwa $2-3$ Zellen tief und 4-7 Zellen breit ist. In solchen Fällen findet man meist, dass als Ersatz für das schwach ausgebildete Collenchym die weiter unten beschriebenen Libriformbrücken der Strangscheide besonders stark entwickelt sind, so bei den Caucalis- und Torilis-Species, Crithmum maritimum, Coriandrum sativum ect.

Das von dieser Form des Collenchyms abweichende Vorkommen bei dem Tribus Hydrocotyleae ist schon oben ausführlich beschrieben worden. Es fehlt vollständig bei Dimetopia isocarpa und D. Walkeri.

Bei Mulinum ferosa wird das Collenchym ersetzt durch einen mächtigen, nach aussen und innen scharf abgegrenzten Mantel verholzter, weitlumiger, einfach getüpfelter Zellen, welche Interzellularräume zwischen sich lassen, und sich auf dem Längsschnitt als vertikal gestreckte, meist spitz zulaufende, doch auch zuweilen mit horizontalen Querwänden versehene Elemente darbieten. Die Zellen der innersten Schicht dieses Holzmantels sind meist von geringerem Durchmesser, die Wände sind aber stärker verdickt und gleichen den charakteristischen Fasern des Hartbastes. Fig. 4.

Bei dem Tribus Saniculeae findet man auch die charakteristischen, collenchymatischen Platten, diese sind aber meist untereinander durch eine einzellige, collenchymatische Schicht verbunden.

Das Rindengewebe bietet wenig Erwähnenswertes, es ist bald mehr, bald weniger ausgebildet. Im allgemeinen unterscheidet man das, zwischen den Collenchymplatten gelegene Chlorophyllparenchym, meist aus tangential gestreckten, nicht besonders grosse Interzellularräume zwischen sich lassenden Zellen bestehend, und das unter diesem und dem Collenchym sich hinstreckende, farblose Rindengewebe. Die Form, in welcher dieses auftritt, ist meist die des Schwammparenchyms, doch ist dasselbe auch zuweilen aus iso- 
diametrischen und dann meist verdickten, einfach getüpfelten Zellen gebildet.

Eine Sonderstellung nimmt hier wieder Mulinum ferosa ein. Dem oben beschriebenen, mächtigen Holzmantel folgt eine zweizellige Schicht verdickter und verkorkter Zellen. Die äusseren sind in der Richtung des Radius gestreckt, die inneren in der Richtung der Tangente. Diese beiden Zellreihen passen genau auf einander, so dass also einer inneren, tangential gestreckten Zelle jedesmal eine äussere, radial gestreckte folgt.

Es ist dies vielleicht ein Specialfall von Endodermis, ähnlich derjenigen bei den epiphitischen Orchideen, Aroideen ect, welche dort die parenchymatische Rinde von der sie umgebenden, luftführenden Hülle abgrenzt. Es wäre dies dann wohl der erste Fall einer überall gleichmässigen, aus einer zweifachen Zellenlage bestehenden Schutzscheide. Dieser Schutzscheide folgt in centripetaler Richtung das Rindengewebe. Es bildet einen mächtigen, gleichmässigen Cylinder kleinlumigen Schwammparenchyms, in welchem unter jedem - weiter unten noch zu besprechenden - Harzgange eine einzige, vollständig isolierte, typische, scharf kantige Sclerenchymfaser liegt, von derselben Beschaffenheit, wie sie sonst als Bastfaser im Hartbast vorkommt, ohne jedoch mit dem Bast in irgend welcher Beziehung zu stehen. Fig. 4.

Bei Ferula ferulago findet sich ausser dem oben beschriebenen, typischen, collenchymatischen Gewebe noch ein zweites Hypoderm, in Form eines mächtigen Ringes verdickter Zellen, welcher nach innen Ausbuchtungen zeigt, in welchen die Gefässbündel des regulären Gefässbündelringes liegen, und von diesen nur durch eine einzellige Schicht auffallend weitlumigen, verdickten, parenchymatischen Gewebes getrennt wird. Dieses zweite Hypoderm besteht aus kolossal verdickten, kreisrunden Zellen, welche nicht dicht in einander ge- 
fügt sind, sondern grosse Interzellularräume zwischen sich lassen. Auf dem Längsschnitt zeigen sich diese Zellen rektangulär gestreckt und einfach getüpfelt. Nach innen geht diese hypodermale Schicht allmählich in das Markgewebe über. Fig. 5 .

Korkbildung wurde nur bei einigen Vertretern des Tribus Hydrocotyleae beobachtet, bei Hydrocotyle villosa, H. flexuosa und Trachymene ovata. Die Initiale liegt stets in der äussersten subepidermalen Schicht, dicht unter der Epidermis. Es bestätigt sich also hier die häufig aufgestellte Behauptung, dass der Entstehungsort des Korkes für die Gattung stets derselbe ist. Der Kork ist stets vielschichtiger Plattenkork und zieht sich unter der vollständig intakten Epidermis in 5 bis 7 zelliger Schicht regelmässig hin. Die Wandstruktur dieser Zellen zeigt nichts Bemerkenswertes, sie sind meist recht derbwandig.

\section{Bast- und Holzkörper.}

Die Gefässbündel, wenigstens die des regulären Gefässbündelringes, sind stets collateral gebaut.

Das Phloëm soll nur der Vollsändigkeit halber mit kurzen Worten erwähnt werden. Die Untersuchung desselben bietet an getrocknetem Herbarmaterial wegen des Eintrocknens und Zusammenschrumpfens des Weichbastes zu grosse Schwierigkeiten gegenüber den unbedeutenden Resultaten, welche für eine vergleichende Anatomie und Systematik ausgenutzt werden können. Es liegt meist in Form einer kleinen Platte vor dem Xylemteil des gewöhnlich geschlossenen Holzkörpers; nur selten bildet es durch interfasciculares Cambium erweitert einen concentrischen Ring, wie bei Hydrocotyle criantha und $H$. villosa. Sehr häufig ist es durch Produktion von Hartbast ausgezeichnet. Dieses liegt dann gewöhnlich am Rande des Phloëms und besteht 
selten nur aus einigen verholzten Bastfasern, wie bei Silaus virescens, meist aber aus einer mehr oder weniger grossen Platte. In den markständigen Bündeln mancher Arten finden sich mitten im Weichbast verholzte Bastfasern vor, so bei Ferula ferulago, Ferula campestris, ganz besonders stark ausgebildet aber bei Peucedanum officinale.

Nicht selten nehmen noch andere sclerotische Elemente an der Bildung des primären und alsdann das Phloëm rings einschliessenden Hartbastringes teil. Dieser Fall zeigt sich besonders ausgeprägt bei allen Species des Tribus Thapsieae und erweist sich also hier von systematischem Werte.

Manchmal tritt der Fall ein, dass derartige Hartbastplatten ohne Übergang in die, die Gefässbündel vereinigende Strangscheide seitlich übergehn, so dass dann der Weichbast wie eine Insel in ringsum verholzten Elementen liegt, so bei Physospermum aequifolium. Geht diese Verschmelzung noch weiter vor, wie in dem äussersten Gefässbündelring von Cachrys crispa, so entsteht eine überall gleich breite Sclerenchymzone, in welcher die Bündel, vollständig eingeschlossen, regelmässig angeordnet sind.

Die konstituierenden Elemente des typischen Hartbastes sind meist sehr englumig und deutlich geschichtet, weniger trifft dies zu bei den zuletzt beschriebenen Fällen (Sclerenchymring), und bei Silaus virescens ist das Lumen auffallend gross.

Bei Pastinaca sativa, P. collina, P. pratensis, Peucedanum verticillare u. a. sind die Bastfasern an der äussersten Grenze des Weichbastes zuweilen kallös verdickt und stärker lichtbrechend; eine Verholzung der Zellwand ist hier eben noch nicht eingetreten.

Die aufbauenden Elemente des Holzkörpers bestehen neben den trachealen Elementen vorwiegend aus verholzten Faserzellen (Libriform- oder Sclerenchymfasern), 
welche mit Ausnahme von Mulinum ferosa nirgend fehlen. Dieselben gehören hauptsächlich der Strangscheide an, welche einmal nach dem Mark hin die äussersten Elemente des Xylems bildet, dann aber, und zwar mit nur wenigen Ausnahmen, die Gefässbündel auf der Xylemseite durch sogn. Libriformbrücken ununterbrochen mit einander verbindet. Hierdurch erhält der Holzkörper das Aussehen eines vollständig geschlossenen Hohlcylinders. Diese Libriformfasern sind meist radial angeordnet und stets einfach getüpfelt, nur in ganz vereinzelten Fällen bei Didiscus coeruleus, Hydrocotyle criantha und $H$. flexuosa beobachtete ich daneben hofgetüpfelte Elemente. In ihrer radialen Ausdehnung variieren diese Libriformbrücken sehr. Da wo das collenchymatische Hypoderm nicht besonders ausgebildet ist, findet man dieselben vornehmlich stark entwickelt und dann nur wenig schmaler als die Gefässbündel selbst, z. B. bei allen Caucalis- und TorilisArten. Anderseits kann ihre Entwickelung auch sehr schwach sein, und besteht die Strangscheide dann nur aus wenigen Zelllagen, so bei allen Helosciadium-Arten, bei Bowlesia tenera, Spananthe radiculosa und Sp. paniculata. Bei den beiden letzten Arten tritt das Xylem als eine schmale Platte von $4-5$ grosslumigen Gefässen, welche radial hintereinander angeordnet sind, in das Mark hinein, während die Libriformbrücke nur aus einer Zelllage nicht mal besonders englumiger aber stets verholzter, einfach getüpfelter Faserzellen besteht und so fast ganz zurücktritt. Nach der Rinde hin schliesst die Strangscheide meist scharf $a b$, während sie in das Mark allmählich übergeht und hier Übergangsformen in Gestalt derbwandiger, langgestreckter, aber abgeplatteter, stets einfach getüpfelter, teilweise verholzter Zellen zeigt.

Diese Strangscheide ist, wie gesagt, für die Umbelliferen typisch und fehlt nur bei wenigen, vornehm- 
lich krautartigen Species, so bei Cicuta virosa, Oenanthe fistulosa, O. phellandrium, Conium maculatum, einigen Hydrocotyle-Arten ect., dann bei manchen Species, die markständige Bündel haben, so bei Ferula communis, Ferula ferulago, Ferula galbanifera, Peucedanum officinale, Pachypleurum cactroides und endlich bei solchen Formen, bei welchen die Hartbastplatten besonders stark entwickelt sind: Laserpitium latifolium, L. Nestleri, L. asperum, Siler trilobum.

Bei diesen verhältnismässig wenigen Ausnahmen, bei welchen die Gefässbündel also durch sogn. primäre Markstrahlen getrennt sind, verhindert die Strangscheide meist eine Verbindung zwischen Mark und Rinde; alsdann fehlen die Markstrahlen meist vollständig. Typische Markstrahlen, welche den ganzen Holzkörper ein- oder mehrreihig, radial gestreckt durchziehen, finden sich nicht sehr häufig. Sie kommen vor bei manchen Arten der Gattungen Xanthosia, Bupleurum, Seseli, Heracleum und Pastinaca. Da wo der Holzkörper durch Aneinanderrücken der Gefässbündel ohne besondere Libriformbrücken zu einem geschlossenen Ring wird, in welchem die Gefässe unregelmässig auf dem ganzen Querschnitt verteilt sind, finden sich ebenfalls Markstrahlen, so bei Hydrocotyle villosa und Trachymene ovata. Die Markstrahlen bieten also hier keine Anhaltspunkte für systematische Zwecke.

Die trachealen Elemente des Holzes sind auch meist in radiale Strahlen angeordnet. Zwischen den Tracheen, den eigentlichen Gefässen, und den Libriformfasern kommen vielfach Übergangsformen vor: Tracheiden, die stets wie diese einfach getüpfelt sind. Die Art der Verdickung der Gefässwandungen ist für eine Differentialdiagnose bei der Familie der Umbelliferen von keiner Bedeutung. Es verdient aber hervorgehoben zu werden, dass alle Arten fast die sämtlichen Verdickungsformen von getüpfelter bis zur spiralig und 
ringförmig verdickten Wand aufweisen. Die Hoftüpfel sind spaltenförmig oder kreisrund. Die einfachen Tüpfel sind meist spaltenförmig, oft in horizontaler Richtung bedeutend gestreckt, und führen so zu treppenförmig verdickten Gefässen, oder sie sind abgerundet und einander so nahe gerückt, dass diese Gefässe den netzförmig verdickten sehr ähnlich erscheinen. Weiter dem Marke zu liegen die Spiralgefässe; dieselben zeigen fast bei jeder Art alle vorkommenden Formen: enge, horizontale Windungen in den weiten Gefässen, als auch weite, senkrecht gestellte und dann häufig doppelte Spiralleisten in den engen Gefässen. Die äussersten gegen das Mark vorgeschobenen trachealen Elemente sind durchgängig ringförmig verdickt.

Die von Solereder ${ }^{1}$ ) als besonders wichtig für die Systematik bezeichnete Perforation der Querwände in den Gefässen kann ich nicht bestätigen. Dieselbe ist mit wenigen Ausnahmen einfach und zeigt sich meist als ein gleichmässig verdickter Rand, der nach Resorbtion der Querwand als bezeichnende Grenze der einzelnen Gefässglieder stehen geblieben ist. Sie kommt nur in den Tüpfelgefässen vor und ist entweder bei schräg gestellter Querwand elliptisch, oder bei horizontal gestellter kreisrund. Bei Berula angustfolia konnte ich den Verlauf der Resorbtion sehr schön beobachten. In den weitlumigen, hofgetüpfelten Gefässen standen die ebenfalls hofgetüpfelten Querwände teilweise noch vollständig intakt da, während an andern Stellen dieselben durch mehr oder weniger grosse Öffnungen durchbrochen waren, endlich war auch die Resorbtion bis zu der beschriebenen, horizontalen, kreisrunden Verdickung fortgeschritten.

Leiterförmige Perforation fand ich nur bei Hydro-

$\left.{ }^{1}\right)$ Über den systematischen Wert der Holzstruktur. München 1885. 
cotyle triloba (1 Spange), Carum Carvi (7 - 9 Spangen), Bupleurum tennissimum (5-6 Sp.), Aethusa cynapium (7 Sp.), Oenanthe globulosa (1 Sp.), Peucedanum verticillare (6 Sp.).

Die Gefässbündel des regulären Ringes sind alle collateral gebaut. Ausser diesen kommen aber bei einer Reihe von Species noch markständige Bündel vor, die meist nicht streng collateral gebaut sind. Es entstehen hier durch Verschmelzung zweier oder mehrerer Bündel verschiedene Zusammensetzungen. Die concentrische Zusammensetzung findet sich häufig, so bei Peucedanum officinale Fig. 6, wo ausserdem noch hinzutritt, dass die Mitte von einer mächtigen Platte typischen Hartbastes eingenommen wird, um welche sich dann in concentrischen Kreisen Phloëm und Xylem anordnen. Concentrische Bündel finden sich ferner bei Laserpitium latifolium, L. Nestleri und L. asperum. Diese concentrische Anordnung ist manchmal nicht überall gleichmässig, so dass die mannigfaltigsten Formen entstehen; um das central gelegene Phloëm ist dann nicht gleichmässig Xylem gelagert, sondern es schliesst sich an zwei oder drei Seiten eine grössere oder kleinere Xylemplatte an. Umgekehrt ist es bei Silaus pratensis, wo sich in der Mitte des Markes zwei Stränge mit der Xylemseite an einander lagern und so das Aussehen eines bicollateralen Bündels gewähren. Im allgemeinen stehen diese Bündel aber isoliert im Marke und sind dann wie die regulären gebaut.

Das Vorkommen dieser markständigen Bündel ist für die Gattung nicht constant, sondern ist eine specifische Eigenschaft. Die Orientierung derselben ist auch verschieden, dieselbe kann richtig, umgekehrt oder regellos sein. Auch ist die Anordnung nicht immer dieselbe. Diese Bündel können zu einem zweiten, dritten ect Ring angeordnet sein, oder sie sind über das ganze Mark regellos zerstreut, oder auch teils 
regelmässig teils unregelmässig gestellt. Für die Gattung scheint Orientierung und Anordnung für gewöhnlich konstant zu sein.

Von 7 untersuchten Oenanthe Species fand ich 2 mit markständigen Bündeln. Oenanthe crocata hat auf dem ganzen Querschnitt nur ein umgekehrt orientiertes, markständiges Bündel. Bei Oenanthe annomala sind die umgekehrt orientierten Bündel zu einem zweiten Ringe angeordnet. Coenolophium Fischeri hat nur ein richtig orientiertes, markständiges Bündel. Bei Silaus pratensis besteht ein zweiter Ring richtig orientierter Bündel, und ausserdem die im Centrum des Markes gelegenen, obenbeschriebenen Bündel. Bei Pachypleurum cactroidessind alle Bündel regellos orientiert und angeordnet. Bei Ferula nudicaulis, F. communis und F. granatensis zeigt der Querschnitt einen zweiten Ring richtig orientierter Bündel und zwischen diesen beiden Ringen unregelmässig orientierte, kleinere Bündel. Dasselbe gilt von Cachrys crispa und C. laevigata. Bei Ferula ferulago besteht unregelmässige Orientierung und Anordnung, ebenso bei Peucedanum heterophylla und P. officinale. Bei Peucedanum orioselinum besteht ein zweiter Ring richtig orientierter Bündel und ausserdem sind noch einige Bündel regellos zerstreut. Bei Laserpitium latifolium, L. Nestleri und L. asperum sind die markständigen Bündel regellos angeordnet und meist concentrisch orientiert, Laserpitium alpinum hat nur ein markständiges, umgekehrt orientiertes Bündel. Thapsia villosa zeigt einen zweiten Ring richtig orientierter und ausserdem noch willkürlich orientierte und angeordnete, markständige Bündel. Zuweilen sind diese Bündel auch durch Hartbastbelege ausgezeichnet, so bei Oenanthe crocata, Peucedanum heterophylla, P. officinale ect. Über das Vorkommen von Hartbast bei Ferula ferulago und F. campestris habe ich schon oben ausführlich berichtet.

Nach den übereinstimmenden Urteilen von Joch- 
mann und Reichhardt ${ }^{1}$ ) treten die markständigen Bündel nicht in den Blattstiel aus, sie sind stammeigen. Sie ziehen parallel und senkrecht durch das Internodium, hie und da geteilt und streckenweise vereintläufig; im Knoten anastomosieren sie durch Verbindungsstränge mit einander, und mit den Strängen des Ringes. Von den Knoten-Anastomosen gehen die das nächste Internodium durchziehenden Markbündel ab. Die des untersten Internodiums über der Wurzel setzen sich an die des Ringes daselbst an resp. entspringen von diesen; die des untersten Internodiums eines Astes ebenso, ohne mit denen des Stammes in direkter Continuität zu stehen.

Bei Mulinum ferosa ist noch ein besonderes Vorkommen von Gefässbündeln zu erwähnen. In dem direkt unter der Epidermis verlaufenden Holzmantel zeigen sich zuweilen vier, also quasi rindenständige Bündel, die so gelagert sind, dass sie den vier Ecken eines Quadrats entsprechen. Dieselben sind collateral gebaut und richtig orientiert, nicht sehr gross, aber ringsum von kolossal verdickten Faserzellen umgeben.

Ein ganz auffallendes, in der Litteratur bis jetzt noch nicht erwähntes Vorkommen von Gefässbündeln beobachtete ich bei Siler trilobum Fig. 7. Vereinzelt kommt hier mitten in den stark entwickelten Hartbastplatten ein concentrisches Bündel vor. Dasselbe ist so angeordnet, dass die Mitte von einer Xylemplatte ausgefüllt wird, die von einem concentrischen Ringe typischen Weichbastes umgeben ist.

\section{Das Mark.}

Das Markgewebe nimmt meist $2 / 3$ des ganzen Querschnitts ein. Dieses ist natürlich nicht der Fall, wo eine Resorbtion des Gewebes im Innern stattgefunden hat, wo der Stengel also hohl ist. Bei manchen Hydro-

$\left.{ }^{1}\right)$ De Bary: Vergleichende Anatomie der Vegetationsorgane, pag. 264. 
cotyleae-Species, die ein eng zusammengedrängtes Strangsystem besitzen, ist die Ausbildung des Markes im Vergleich zu den übrigen Gewebsarten ebenfalls eine geringe. Bei Bowlesia tenera, Cicuta virosa und allen Arten der Gattung Helosciadium fehlt das Mark vollständig, und die Gefässbündel ragen nach innen bedeutend über das Grundgewebe hinaus.

Die Form der Markzellen ist auf dem Querschnitt eine rundliche, oder, durch gegenseitigen Druck entstanden, isodiametrisch-polyedrische. In centrifugaler Richtung erleiden dieselben im allgemeinen eine Verringerung ihres Querdurchmessers, bei abnehmender Seitenzahl und beständiger Längsstreckung. Auf dem Längsschnitt sind sie rechteckig und im Centrum ungefähr viermal so lang als breit. Die Markzellen schliessen nicht dicht aneinander, sondern lassen kleine, meist dreieckige Interzellularräume zwischen sich. Nach dem Xylem zu verschwinden dieselben, durch lückenloses Ineinandergreifen der gestreckten, meist vierseitigen Zellen zu einer Markscheide.

Bei Oenanthe phellandrium ist das Grundgewebe, wie bei Wasser- und Sumpfpflanzen häufig beobachet wird, gleichmässig von weiten interzellularem Luftgängen durchzogen.

Die Zellen des Markes sind bei den Umbelliferen stets verdickt und einfach getüpfelt. Die Verdickung ist aber nicht überall gleich ausgeprägt. Als Gegensätze könnten hier beispielsweise Trochiscanthes nodiflorus und Libanotis montana angeführt werden. Die spärlich getüpfelten Markzellen der ersten Art könnten im Vergleich zu denjenigen der zweiten geradezu als zartwandig gelten.

Inhalt führen die Markzellen höchst selten, und hätten wir es demnach mit dem von A. Gris ${ }^{1}$ ) bezeichnetem moelle inerte zu thun.

1) Mémoire sur la moelle des plantes ligneuses. Nouvelles Archives du Mus. d' hist. nat. Paris 1870, p. 201. 
Kalkoxalat fand ich nur bei wenigen Arten: bei IIydrocotyle virgata und $H$. villosa in geringer Menge in Form von Krystalldrusen, sehr verbreitet jedoch im ganzen Grundgewebe in derselben Form bei Eryngium Barrelieri und Mulinum ferosa; die Drusen füllen hier die Zelle ganz aus und sind deshalb nach De Bary als Krystallschläuche anzusehen. Bei Mulinum finden sich diese Krystallschläuche reihenweise über und neben einander angeordnet.

Ausser diesen Krystallen fand ich noch krystallinische Bildungen bei Seseli glaucum, S. montanum, S. coloratum, Peucedanum arenarium und Coriandrum sativum. Diese krystallinische Bildungen konnte ich nicht identificieren. Gegen verdünnte Salz- und Schwefelsäure verhielten sie sich indifferent, lösten sich aber sehr leicht in Kalilauge. Concentrierte Schwefelsäure löste sie unter Hinterlassung eines gelben Rückstandes (Verkohlung). Es lagen hier demnach wahrscheinlich die beim Einlegen der Pflanzen in Alkohol häufig sich ausscheidenden, seidenglänzenden Sphaerite stickstoffhaltiger, organischer Substanzen vor, wie Asparagin, Leucin und Tyrosin.

Die für die Umbelliferen so charakteristischen schizogenen mit Harz gefüllten Interzellularräume sind im ganzen Gewebe zerstreut. Die Annahme De Bary's, dass dieselben bei allen Arten vorkämen, kann ich vollauf bestätigen. Stets ist ein Harzgang vor dem Gefässbündel gelegen, zwischen diesem und der Collenchymplatte. Nach den Untersuchungen Müller's ${ }^{1}$ ) finden sich dieselben sehr häufig auch im Phloëm vor. Das Mark ist oft vollständig frei von Harzgängen.

1) C. Müller: Über phloëmständige Sekretkanäle bei den Umbelliferen: Bericht der deutschen botanisc،en Gesellschaft, Bd. VI, pag. 20. 
Anderseits ist jedoch das Vorkommen derselben im Marke für manche Gattungen typisch, so bei Heracleum und Peucedanum und dem ganzen Tribus Thapsieae.

\section{Specieller Teil.}

Wie schon in der Einleitung hervorgehoben wurde, reichen die anatomischen Merkmale der Achse nicht aus, um die einzelnen Triben zu charakterisieren. Es zeigen die Untersuchungen aber immerhin, dass morphologische und anatomische Merkmale bei gewissen Gruppen der Umbelliferen zusammenfallen, dass also die histologischen Charaktere des Stengels zur Diagnose der Triben verwendet werden können.

In Betreff der Begrenzung der Gattungen und Arten bin ich überall De Candolle's Prodromus gefolgt. Die Einteilung in Unterfamilien und Triben entnahm ich Thomé's Flora von Deutschland.

Die untersuchten Pflanzen sind folgende:

\section{Heterosciadieae.}

Hydrocotyleae:

Hydrocotyle vulgaris L., H. virgata L., H. villosa L., H. triloba Thunb, H. flexuosa L.

Dimetopia isocarpa, D. Walkeri.

Didiscus coeruleus D. C.

Trachymene stricta, T. ovata Rudg.

Xanthosia rotundifolia, X. pilosa.

Saniculeae:

Sanicula canadensis L.

Dondia epipactis Neck.

Astrantia heliborifolia Salisb., Ast. major L., Ast. alpinia gracilis Schultz.

Eryngium Barrelieri Boiss. 
Mulineae:

Mulinum ferosa.

Bowlesia tenera.

Spananthe paniculata.

\section{Haplozygieae.}

Ses elinea e.

Oenanthe apiifolia Brot., Oe. annomala Coss., Oe. globulosa L., Oe. crocata L., Oe. fistulosa L., Oe. phellandrium Lam.

Aethusa elata Friedl., Ae. cynapium L.

Foeniculum officinale All.

Kundmannia sicula Scop.

Seseli coloratum Erch.. S. montanum L., S. Gouani Koch, S. campestre Bess., S. glaucum Jacq., S. libanotis, S. hippomarathrum L.

Wallhrothia tenuifolia D. C.

Libanotis montana Koch.

Coenolophium Fischeri Koch.

Trochiscanthes nodiflorus Koch.

Athamantha vestina Kern.

Ligusticum pyrenaeum Gou., L. obtusifolium Horn, L. carniolicum Host.

Silaus virescens Griseb., S. pratensis Bess.

Meum athamanticum Jacq., M. mutellina Gaertn., M. nevadense Bess.

Crithmum maritinum L.

Pachypleurum cactroides.

Ammineae:

Cicuta virosa L., C. orientalis.

Zizia latifolia Koch, Z. integerrima D. C., Zizia aurea Koch.

Apium graveolens L.

Petroselinum sativum Hofm.

Trinia vulgaris Hofm., T. Henningii Hofm. 
Helosciadium nodiflorum Koch, H. innundatum L., H. repens $L$.

Discopleura capillacea D. C.

Ptychotis Thumbalii Jord., P. heterophylla Koch.

Ammi majus L.

Sison amomum L.

Aegopodium podagraria L.

Falcaria latifolia Koch, F. Rivini Bernh.

Carum carvi L., C. bulbocastanum Koch.

Bunium virescens D. C., B. montanum Koch.

Conopodium pyrenaicum Mieg.

Pimpinella dichotoma L., P. saxifraga L., P. rotundifolia Bieb., P. magna Wild.

Rentera rigidula Boiss.

Berula augustifolia L.

Sium latifolium L., S. sisarum L., S. siculum L.

Bupleurum junceum L., B. petriculum Gdgr., B. tennissimum L., B. aureum.

\section{Peucedaneae.}

Ferula nudicaulis Spreng., F. campestris Bess., F. granatensis, F. communis L., F. silvatica Bess., F. ferulago L.

Peucedanum orioselinum Moench., P. arenariumW. K., P. Chabraei Rehb., P. officinale, P. heterophyllum Vis., P. verticillare Koch., P. palustre.

Bubon glaucus Sp.

Anethum graveolens L.

Pastinaca sativa L،, P. Fleischmannii, P. collina Jor., P. pratensis Bord.

Heracleum spondylium L., H. alpinum I., H. montanum Schl., H. sibiricum L.

Tordylium apulum Riv., T. maximum L.

Cumium cymium. 
Angeliceae:

Levisticum vulgare Rehb.

Selinum palustre L.

Ostericum palustre Bess.

Angelica offic. silvestris L., A. pratensis Bieb., A. montana Schleh.

Archangelica offic. Hofm.

Scandineae:

Scandix pecten veneris $\mathrm{L}$.

Anthriscus trictosperma Schult., A. nitidus Wahlb., A. alpestris Wimmer, A. vulgaris Pers., A. cerefolium L. Chaerophyllum procumbens Lam., Ch. elegans Gaud., Ch. magallense Ten., Ch. hirsutum L., Ch. cicutarium Vill., Ch. aureum L., Ch. temulum L.

Meloposperum cicutarium Koch.

Myrrhys odorata Scop.

Smyrneae:

Cachrys crispa Pers., C. laevigata L.

Conium maculatum L.

Pleurospermum austriacum Hofm.

Malabaila yolaka.

Smyrnium perfoliatum Mill., Sm. olusatrum L.

\section{Diplozygieae.}

Thapsieae:

Thapsia villosa $\mathrm{L}$.

Laserpitium siler L., Ls. asperum Crantz, Ls. peucedanoides L., Ls. pruthenicum L., Ls. Nestleri Will. Ls. latifolium L., Ls. luteolum Gaud., Ls. nitidum Ztsch. Melanoselinum Hoffmannii.

\section{Silerineae:}

Siler trilobum. 
Daucinaeae:

Orlaya grandiflora Hofmannii.

Daucus muricatus Desf., D. setulosus Guss., D. hispidus Desf.

\section{Caucalineae:}

Caucalis daucoides L., orientalis L., C. muricata L. Turgenia latifolia L.

Torilis helvetica Gesn., T. heterophylla Gurz., T. nodosa Gaertn., T. infesta Koch.

Coriandreae:

Coriandrum sativum L.

Bifora radians Bieb.

Anatomische Verwandtschaft morphologischer Gruppen.

Eingangs erwähnte ich schon, dass man nicht mit Unrecht in jüngster Zeit die Einteilung in die Unterfamilien Orthospermeae, Campylospermeae und Coelospermeae habe fallen gelassen und statt dieser die Gruppierung in Heterosciadieae, Haplozygieae und Diplozygieae eingeführt habe. Die Unterfamilie Heterosciadieae, die morphologisch sich dadurch von den beiden andern unterscheidet, dass ihr Blütenstand die umbella simplex ist im Gegensatz zur umbella composita der andern, und ihre Früchtchen keine Fruchtträger und keine Ölstriemen besitzen, zeigen auch in anatomischer Beziehung vielfach, dass sie die weniger hoch differenzierten Formen umfassen; sie bilden die Übergangsstufe zu den höchsten, typischen Formen.

Die Heterosciadieae umfassen die Triben Hydrocotyleae, Saniculeae und Mulineae. Da die Hydrocotyleae meist Wasserpflanzen sind, benötigen sie als solche auch 
nicht der mechanischen Elemente, welche die Biegungsfestigkeit erhöhen, die vorzugsweise aussen nnd bei den Umbelliferen in vorspringenden Kanten (Collenchymplatte) angelegt sind. Das Skelettsystem ist mehr central in ein eng zusammengedrängtes Strangsystem, welches noch durch Hartbastplatten verstärkt ist, angeordnet, wo es ohne die Seitenbewegungen zu beeinträchtigen seine volle Zugfestigkeit entfalten kann. Es müssen diese anatomischen Verhältnisse also als Funktion der äusseren Lebensbedingungen aufgefasst werden. Das Hypoderm ist deshalb nur gering in Gestalt einer einzelligen, collenchymatischen, subepidermalen Schicht ausgebildet.

Bei den letzten Formen dieses Tribus: Xanthosia rotundifolia und $\mathrm{X}$. pilosa, die schon ausgesprochen terresterischen Charakter haben, ist dieses Hypoderm schon durch sclerotische Elemente verstärkt, welche entweder in dieser Collenchymschicht liegen oder vor dieselbe gelagert sind.

Der Tribus Saniculeae schliesst sich dem vorigen an. Die Ausbildung des Hypoderms nähert sich successive demjenigen der typischen Umbelliferen. Den Gefässbündeln gegenüber liegt hier stets eine Collenchymplatte, die aber, die Verwandtschaft mit dem Tribus Hydrocotyleae dokumentierend, durch eine subepidermale Collenchymschicht mit einander verbunden sind. Diese Schicht hat vielfach eine grosse Ausdehnung, so bei Astrantia major, den monocotylenähnlichen und vielen netzaderigen Eryngien. Bei den letzteren geht aber der Übergang zu den typischen Rindenverhältnissen der Umbelliferen vor sich, hier fehlt plötzlich die verbindende collenchymatische Schicht, so bei Eryngium Barrelieri. Ein weiteres Unterscheidungsmerkmal den Hydrocotyleen gegenüber besitzen die Saniculeen in dem vollständigen Mangel an Hartbast. 
Die Gattung Bowlesia, die von Endlicher und De Candolle noch zu den Hydrocotyleen gerechnet wurde, scheidet Engler in seinem Syllabus aus dem Tribus aus und stellt sie zu dem Tribus Mulineae. Es rechtfertigt sich dies auch aus anatomischen Gründen. Bei Bowlesia ist das Hypoderm das der typischen Umbelliferen und stimmt darin mit der Gattung Spananthe überein. Überhaupt zeigt das Querschnittsbild dieser beiden Gattungen bis in die kleinsten Details eine grosse Übereinstimmung.

Aus der successiven Entwickelung des Hypoderms, das bei den Mulineen seine Höhe erreicht, und welches für sämtliche nun folgenden Gattungen konstant ist, lässt es sich wohl rechtfertigen den Tribus Mulineae statt als zweiten als dritten in der Reihe hinzustellen.

Die Gattung Mulinum, die dem ganzen Tribus den Namen gegeben hat, steht anatomisch betrachtet vollständig isoliert da; sie schliesst sich nach keiner Richtung den morphologisch verwandten Gattungen an. Im Vergleich zu den übrigen Dicotyledonen und speciell den Umbelliferen setzt sich ihr ganzer anatomischer Bau aus lauter Abnormitäten zusammen. Nur die schön ausgebildeten, in Rinde und Mark verlaufenden, zu zwei concentrischen Ringen angeordneten Harzgänge erinnern an eine verwandtschaftliche Beziehung zu der Familie.

Bei den nun folgenden Triben fehlen scharf begrenzte, durchgreifende Unterscheidungsmerkmale in den anatomischen Verhältnissen der Achse.

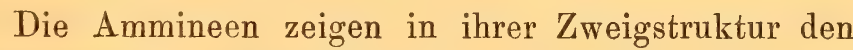
allgemeinen Umbelliferentypus, doch könnte das Fehlen von Hartbast, ausgenommen Rentera rigidula, und der vollständige Mangel markständiger Bündel als diagnostisches Merkmal Verwertung finden, im Gegensatz zu den Seselineen, wo Hartbast stets auftritt und markständige Bündel mehrfach vorkommen. 
Der Tribus Peucedaneae ist durch markständige Bündel besonders oft ausgezeichnet.

Bei dem Tribus Thapsieae dürften vielleicht die stets vorkommen Harzgänge im Mark und die ungemein grossen, oben beschriebenen Hartbastplatten von systematischer Bedeutung sein.

Er stimmt hierin mit dem Tribus Silerineae überein, und es erklärt sich so die verwandtschaftliche Beziehung der beiden Triben auch anatomisch.

Die morphologisch nahestehenden Triben Caucalineae und Coriandreae sind beide durch kolossale Entwickelung der Strangscheide und verhältnismässig geringer Ausbildung des Collenchyms ausgezeichnet. 


\section{Lebenslauf.}

Ich, Friedrich, Carl van Noenen, Sohn des Kaufmannes R. van Noenen, katholischer Konfession, bin geboren am 25. November 1868 zu Geldern, besuchte hier die Elementar- und später die Rektoratschule und kam 1881 nach Aachen, wo ich das Realgymnasium bis 1886 besuchte. Darauf begann ich meine pharmazeutische Laufbahn, welche ich nach bestandener Staatsprüfung in Münster im November 1893 abschloss. Das dem Examen folgende Semester studierte ich in Münster an der Kgl. Akademie Nahrungmittelchemie und die beiden weitern Semester in Erlangen Naturwissenschaften, wo ich am 30. Januar 1895 promovierte. 


\section{Erklärung der Figuren.}

Fig. 1 und 2.

Xanthosia rotundifolia und X. pilosa.

a) Collenchym, b) Sclerenchymfasern.

Fig. 3.

Peucedanum Chabraei.

a) Warzenähnliche Fortsätze der Cuticula.

Fig. 4 .

Mulinum ferosa.

a) Zweischichtige Epidermis,

b) Holzmantel,

c) zweischichtige Endodermis,

d) Rindengewebe,

e) Harzgänge,

f) Phloëm,

g) Xylem,

h) Sclerenchymfaser.

Fig. 5.

Ferula ferulago.

a) Erstes Hypoderm (Collenchym),

b) zweites Hypoderm,

c) dickwandige, parenchymatische Schicht,

d) Hartbastplatte.

Fig. 6.

Schematischer Querchnitt von Peucedanum officinale.

a) Collenchym,

b) Harzgänge,

c) Phloëm,

d) Xylem.

Fig. 7.

Siler trilobum.

A) Reguläre Gefässbündel.

a) Gefässbündel innerhalb einer Hartbastplatte. 

Fig. 1.

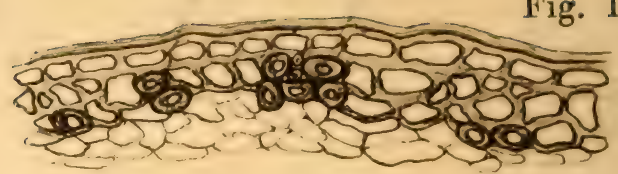

Fig. 2.

Fig. 3.

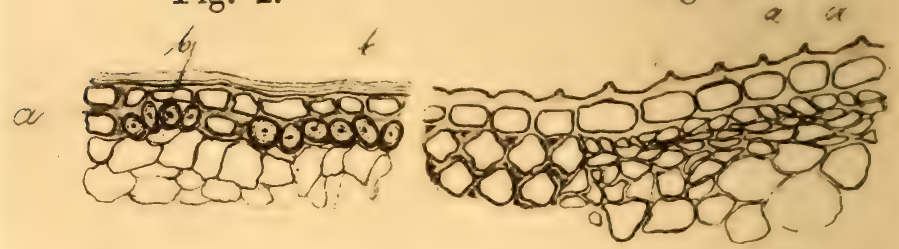

Fig. 5.

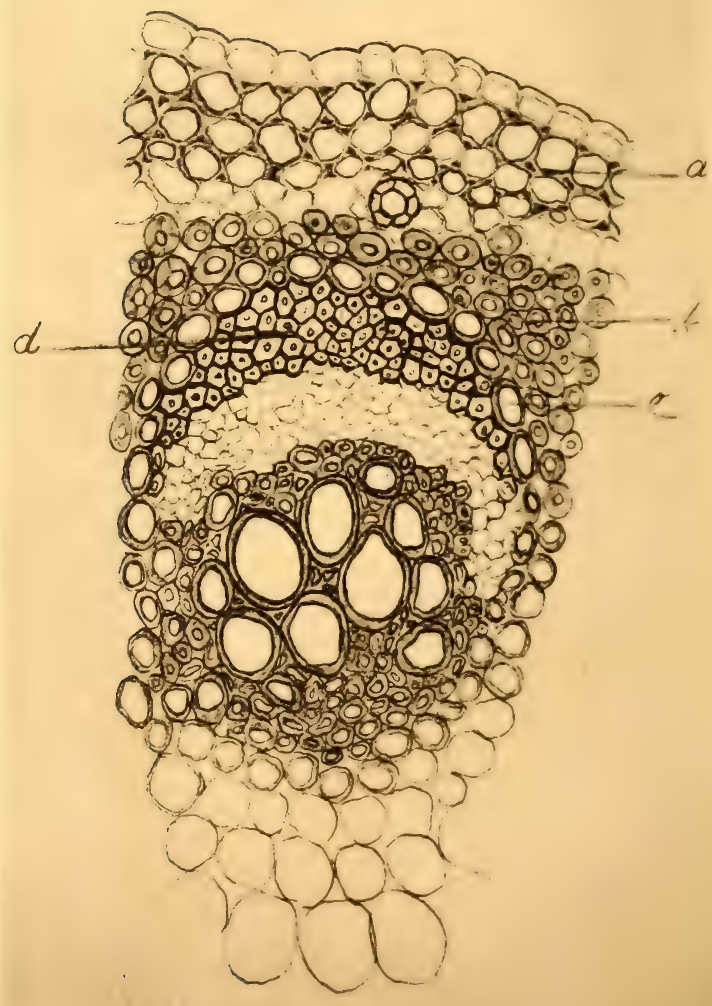



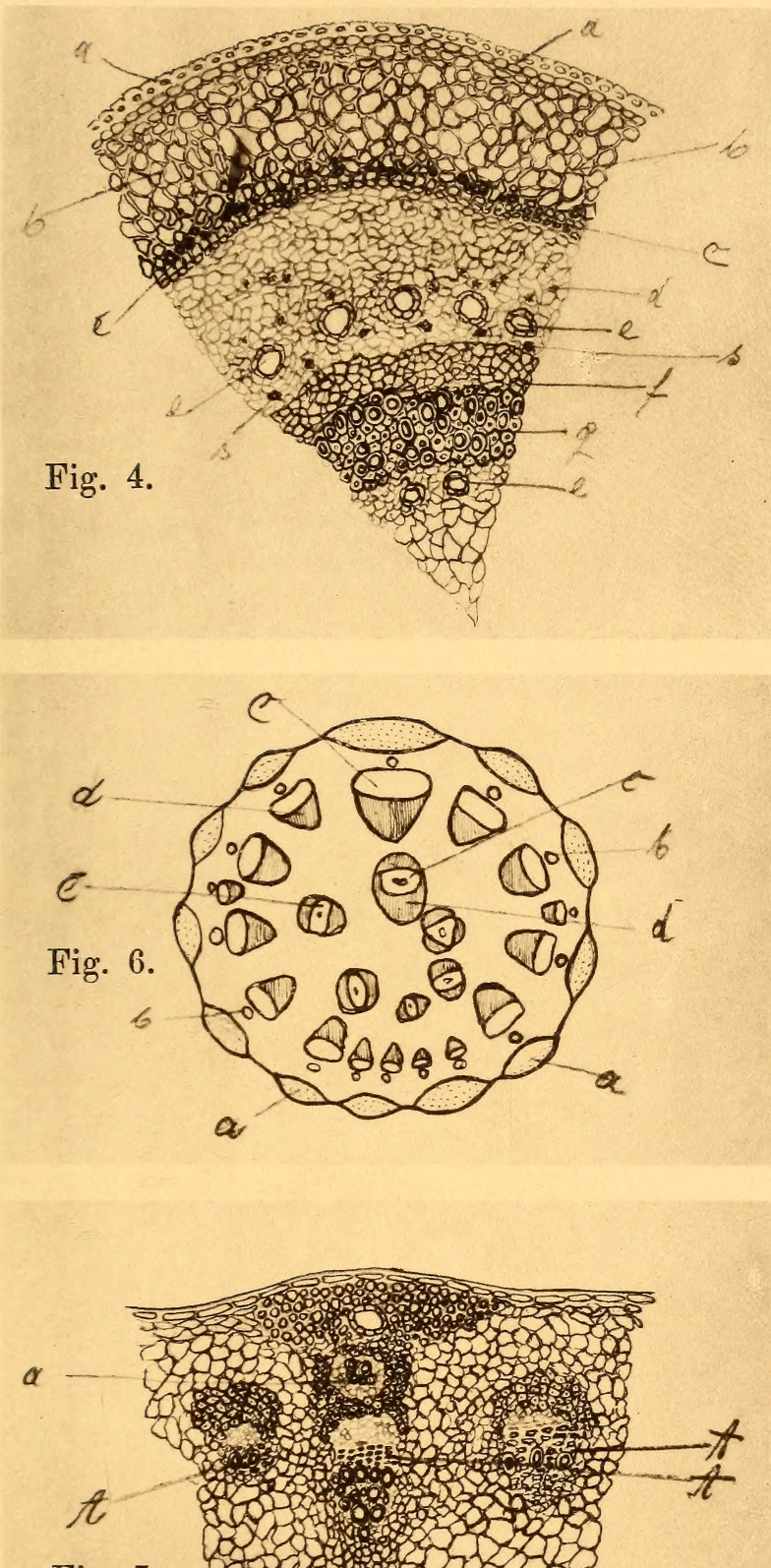

Fig. 7

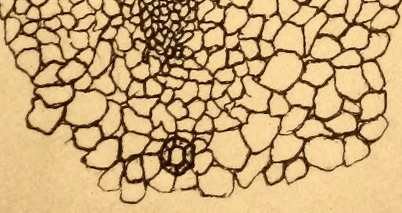



